II Congresso Brasileiro de

Fluidodinâmica Computacional

27 a 29 de Junho de 2018

Rio de Janeiro, RJ

\title{
EFFECTS OF UNIFORM MAGNETIC FIELD ON THE NON- NEWTONIAN HEMODYNAMICS OF A SACCULAR BRAIN ANEURYSM 3D MODEL.
}

\author{
M. CARDONA ${ }^{1}$, A. RIVERA ${ }^{1}$, A. BENAVIDES ${ }^{2}$ and J. RAMIREZ ${ }^{3}$ \\ ${ }^{1}$ Universidad Nacional de Colombia, Mechanical Engineer Curricular Area \\ ${ }^{2}$ Assistant professor, Universidad Nacional de Colombia, Mechanical Engineer Department \\ ${ }^{3}$ Associate professor, Universidad Nacional de Colombia, Mechanical Engineer Department \\ ${ }^{1}$ E-mail: mecardonata@unal.edu.co
}

\begin{abstract}
The three-dimensional computational model of a simplified saccular brain aneurysm is used to study the time-dependent hemodynamics under the effect of an external magnetic field. The model takes into account the NonNewtonian behavior of the blood to compute the velocity field and wall shear stress (WSS). The intensity of the magnetic field is varied so as to evaluate its effect on the velocity and WSS. Results at systole phase $(0.18 \mathrm{~s})$ show that under the action of an applied external uniform magnetic field, the velocity of the blood flow decreases while the temporal variation of WSS increases in the neck of the aneurysm. In addition, flow is redirected to the bulge creating a stagnant fluid at the aneurysm neck.
\end{abstract}

\section{INTRODUCTION}

The tissue regeneration at the neck of saccular brain aneurysm has been previously investigated, as a treatment that enable thrombogenic occlusion. It has been proposed to use magnetic fields to recruit endothelial cells present in the blood to the aneurysm neck region (Allain et al. 2013). However, to date there is a paucity of understanding about the disturbed flow behavior that includes the complex hemodynamic environment of the blood flow under magnetic fields (Mirzababaei et al. 2017). However, this intricate biological flows can be studied by use of Computational Fluid Dynamics (CFD).

Alimohamadi et al, (2013), have presented a CFD analysis on a 2D idealized saccular aneurysm model in presence of an external uniform magnetic field using Newtonian behavior. However, it is known that rheological properties of blood can significantly affect the flow when magnetic studies are performed, making it necessary to include Non-Newtonian blood constitutive models. The aim of the present study is to describe the effects of applying uniform magnetic fields on the behavior of the blood flow inside the sac of an idealized intracranial wide-neck saccular aneurysm under physiologically realistic pulsatile conditions in a non-Newtonian CFD three-dimensional model.

\section{METHODS}

\subsection{Model geometry}




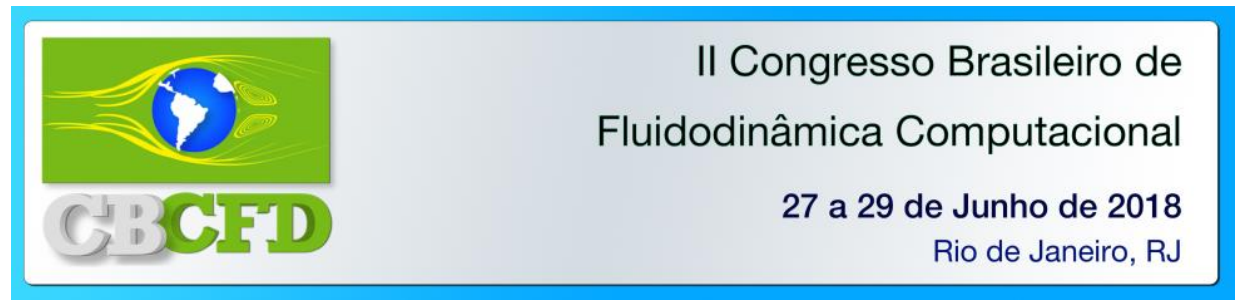

As presented in Figure 1, an idealized 3D model of a Saccular Brain Aneurysm of the anterior communicating artery ( $\mathrm{ACoA}$ ) with a curvature of $60^{\circ}$ is reconstructed, taking into account the most common location of the pathology (Xu et al. 2015). Both perpendicular height and neck diameter are $5 \mathrm{~mm}$ and the aspect ratio (depth/neck width) of 1 . The length $\mathrm{L}$ and diameter $\mathrm{D}$ of the parent vessel was $30 \mathrm{~mm}$ and $4 \mathrm{~mm}$ respectively. To ensure that the flow profile is fully developed, the proximal section of the parent artery is set to 10D.

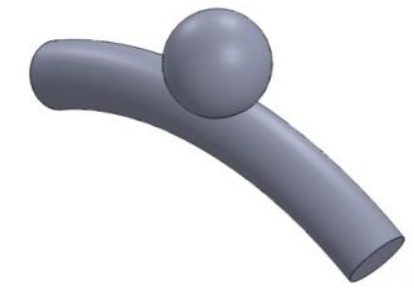

Figure 1. Idealized 3D model of a Saccular Brain Aneurysm

\subsection{Materials and Methods}

Navier-Stokes equations are solved to simulate the laminar, transient and incompressible blood flow, coupled with ferrohydrodynamic (FHD) equations. In addition to continuity Eq. (1) where $u$ is velocity, the magnetization force acting on the fluid is included in the momentum $\mathrm{Eq}$ (2) can be expressed in terms of magnetization $M$, magnetic permeability of vacuum $\mu_{o}$ and the applied magnetic field $H$.

$\nabla \cdot u=0$

$\frac{\partial u}{\partial t}+(u \cdot \nabla) u=\frac{1}{\rho}\left[-\nabla P+\eta \nabla^{2} u+\mu_{0}(M \cdot \nabla) H\right]$

$P$ is Pressure, the density of blood $\rho$ is set to $1050 \mathrm{~kg} / \mathrm{m}^{3}$, magnetic permeability $4 \pi \times 10^{-7} \mathrm{H} / \mathrm{m}$ and a Non-Newtonian rheological behavior is set. Herschel-Bulkley model was considered for the dynamic viscosity $\mu_{f}$ that varies according to Eq (3). Where $k=$ $8,9721 \cdot 10^{-3} \mathrm{~N} s^{n} / m^{2}$ is the Consistency Index, $n=0.8601$ is the Power-Law Index and $\tau_{o}=0.0175 \mathrm{~N} / \mathrm{m}^{2}$ is Yield Stress Threshold (Valencia et al. 2008).

$$
\eta=k \dot{\gamma}^{n-1}+\left(\tau_{0} / \dot{\gamma}\right)
$$

Boundary conditions: Pulsatile waveform for the inlet velocity condition in the fluid domain reported by (Valencia et al. 2008) is specified. Each pulse took of 1 second and the maximum velocity is $1.5 \mathrm{~m} / \mathrm{s}$ at 0.18 seconds in accordance with systole. The vessel wall is assumed rigid and as an insulating state under the applied magnetic field. The outlet is modeled as an open boundary with zero static reference pressure since the model is extended $10 \mathrm{D}$ after the domain of interest. No-slip condition is set on the vessel wall.

Numerical method: The commercial software ANSYS Fluent version 17.1 (ANSYS Inc 2018) is used to perform the numerical simulation using the SIMPLEC segregated solution algorithm. In order to compare the effects of the magnetic field in the flow patterns, different magnetic fields $(0.1,0.5$ and 1 Tesla) in the positive $Y$ axis are implemented. The magnetic field acts on the whole segment of the aneurysm blood vessel. After a sensitivity 


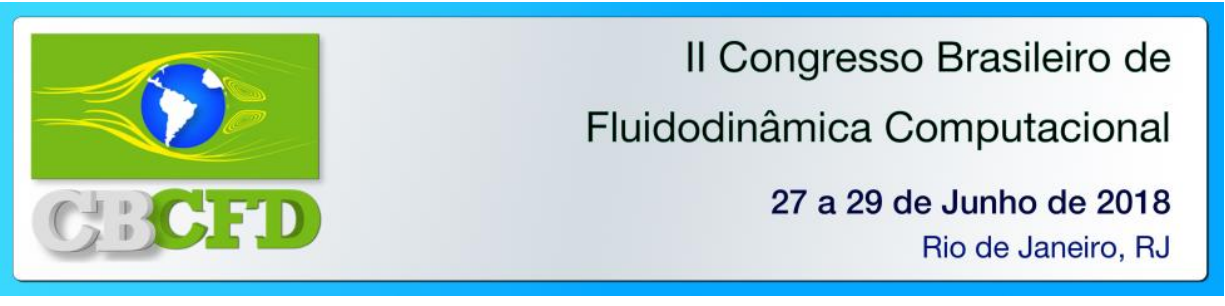

analysis, the spatial discretization of the domain is set to 291705 tetrahedral and hexahedral elements. With $0.005 \mathrm{~s}$ time steps and 100 maximum iterations, the model converges and residual levels drop below 1e-3. The magnetic effects were included in the model using the Magnetic Hydrodynamics module (MHD) in ANSYS Fluent and three consecutive pulsatile flow cycles are run in each simulation to allow the decay of initial transients. Results of the third cycle are presented below.

\section{RESULTS AND DISCUSSION}

Velocity contours at different magnetic field $\mathrm{B}=0,0.1,0.5$ and $1 \mathrm{~T}$ are plotted at systole peak in the third pulse $(\mathrm{t}=2,18 \mathrm{~s})$ using two different planes: Figure 2 (a-d) shows velocity contours at the center plane $\mathrm{xy}, \mathrm{z}=0$ and $(\mathrm{e}-\mathrm{h})$ the results in the cross-section area at plane $y z, x=0$ using the same scale.

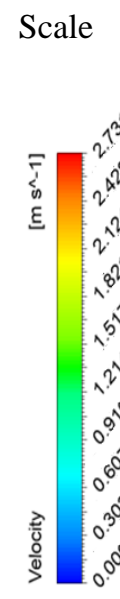

(a) without Magnetism

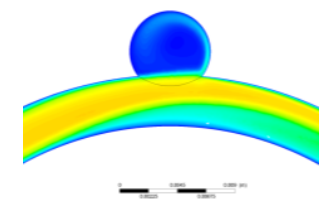

(e)

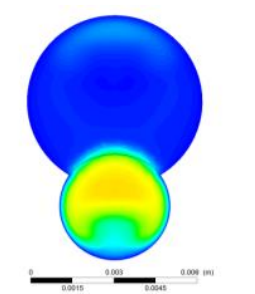

(b) $0.1 \mathrm{~T}$

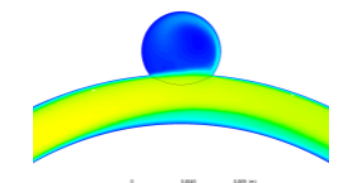

(f)

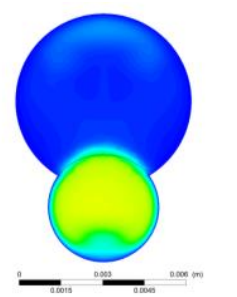

(c) $0.5 \mathrm{~T}$

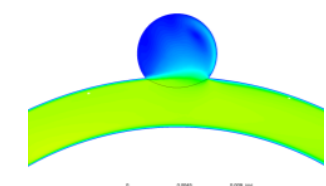

(g)

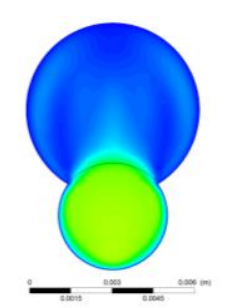

(d) $1 \mathrm{~T}$

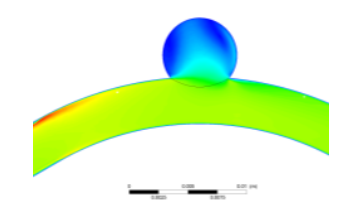

(h)

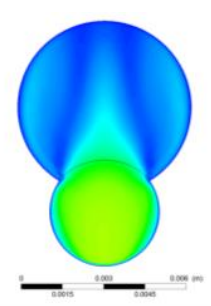

Figure 2 Velocity contours at different magnetic fields, center plane (a-d) and cross-section area (e-h).

As presented in Figure 2, the external magnetic field change the direction of velocity field where the blood flow is pushed strongly in the direction of the magnetic field, making possible to accumulate blood in the aneurysm neck where the tunica media is missing. In addition, it is shown that the velocity magnitude decreases when the intensity of the magnetic field increases, creating vortexes that help to staunch the ferrofluid flow.

The use of idealized geometric models are still a crucial strategy to study Multiphysic phenomenon since it allows to isolate the effect of geometry on the overall hemodynamics analysis (Mirzababaei et al. 2017). As presented in Figure 3 a large magnetic fields promote an increase on the temporal variation of WSS in the pulsatile environment of the nonNewtonian behavior. The effect is most notable in the systolic phase, although the curves have a slightly different behavior. This increment is explained by the abrupt change of velocity that occurs at the distal neck region (point $\mathrm{A}$ ), where the inflow impacts. The results obtained with $B=0 T$ was used to compare the order of magnitude with the results presented by (Xu et al. 2015). 


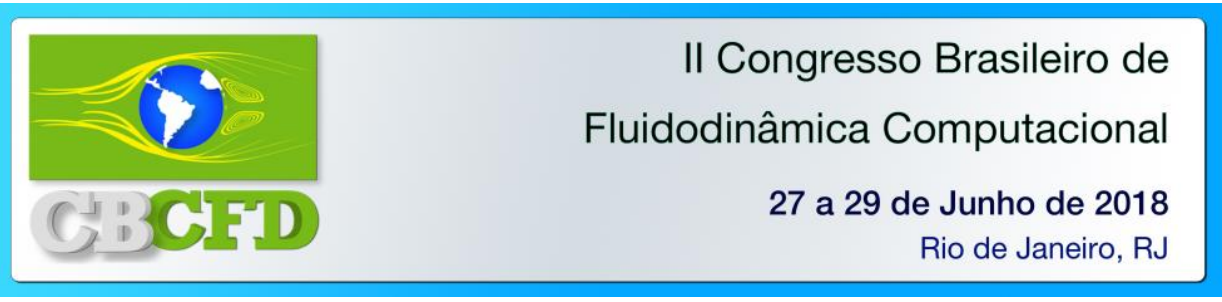

(a) Point of analysis

(b) WSS [Pa] vs t[s]
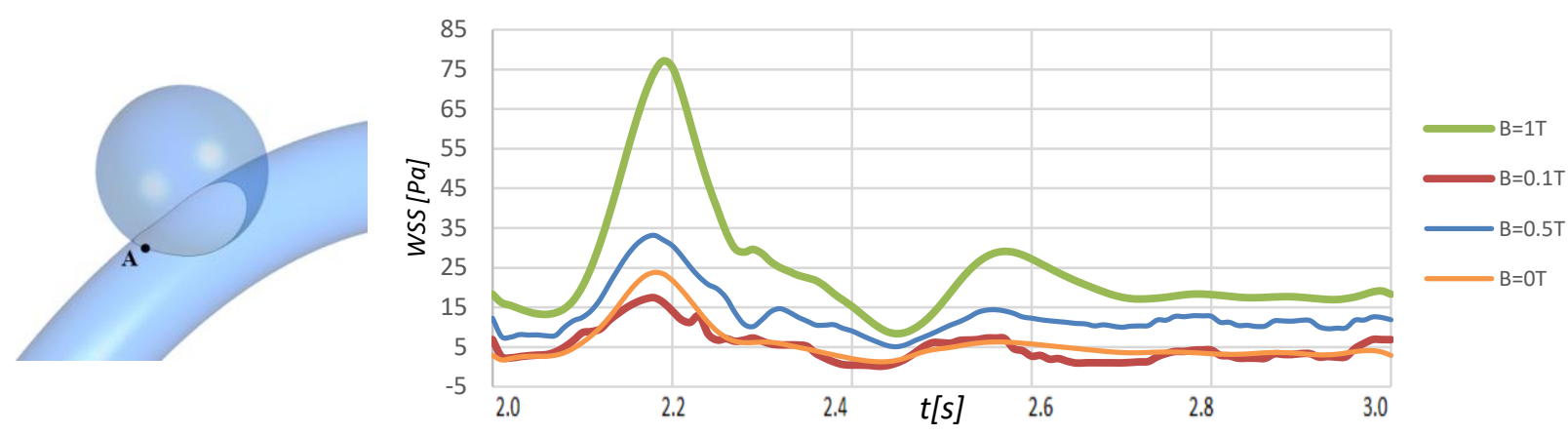

Figure 3 Temporal evolution of (b) WSS at the characteristic point (a) for different magnetic field magnitudes.

\section{CONCLUSIONS}

Under the action of the applied external uniform magnetic field, the velocity of the blood flow decreases and its direction is redirected to the bulge creating a stagnant fluid at the aneurysm neck. This occurrence provides an appropriate condition for endothelial cells recruitment on a specific location, since blood acts as carrier fluid.

In addition, increment in the pulsatile wall shear stress and Pressure at the neck is desirable since it promotes the cells orientation. The presented CFD model gives relevant elements that allows to understand the effect of applying a uniform external magnetic field for cells recruitment. Further realistic models are suggested to consider the effect of the pulsatile environment on the wall vessels and its effect on the disturbed blood flow.

\section{REFERENCES}

ALIMOHAMADI, H., IMANI, M. \& SHOJAEIZADEH, M,. Computational analysis of pulsatile biofluid in a locally expanded vessel under the action of magnetic field. Advances in Applied Science Research, 4(6), pp.96-103, 2013.

ALLAIN, J.P., TIGNO, T. \& ARMONDA, R.,. Nanotechnology for Cerebral Aneurysm Treatment, The Textbook of Nanoneuroscience and Nanoneurosurgery Babak Kateb and John D, 2013.

ANSYS Inc, Version 16. ANSYS® Academic Research Mechanical.

MIRZABABAEI, S.N. GORJI, TAHEREH B, BAOU, M, GORJI-BANDPY, M, FATOURAEE, NASSER., Investigation of magnetic nanoparticle targeting in a simplified model of small vessel aneurysm. Journal of Magnetism and Magnetic Materials, 426, pp.126-131, 2017.

VALENCIA, A, MORALES, H, RIVERA, R, BRAVO, E, GALVEZ, M,. Blood flow dynamics in patient-specific cerebral aneurysm models: the relationship between wall shear stress and aneurysm area index. Medical engineering \& physics, 30(3), pp.329-40, 2008.

XU, J. WU, Z, YU, Y, LV, N., Combined Effects of Flow Diverting Strategies and Parent Artery Curvature on Aneurysmal Hemodynamics: A CFD Study J. Sznitman, ed. PLOS ONE, 10(9), p.e0138648, 2015. 